\title{
Threonine Dehydratase Deficiency : a Probable Cause of Non-ketotic Hyperglycinaemia
}

\author{
I. KRIEGER \\ The Department of Pediatrics, Wayne State University, Children's Hospital of Michigan, Detroit, MI, \\ USA
}

F. BOOTH

Winnipeg Children's Hospital, Manitoba, Canada

\begin{abstract}
A patient with classical symptoms of non-ketotic hyperglycinaemia (NKH) is presented. Threonine dehydratase was undetectable in a liver autopsy specimen, which was obtained within $1 \mathrm{~h}$ of death and immediately frozen at $-70^{\circ} \mathrm{C}$. Activities of four marker enzymes were normal. This represents the first documentation of an inborn error of threonine metabolism and a new explanation of $\mathrm{NKH}$.
\end{abstract}

Recently we made an observation in a patient with NKH (McKusick 23830) which suggested that a disorder of threonine metabolism is the cause of this disease. Since the manifestations of NKH have been attributed to high glycine concentrations in the central nervous system, (CNS), and since threonine is a precursor of glycine in CNS (Maher and Wurtman, 1980), we treated two infants with a threonine-free formula for 1 week (Krieger and Nigro, 1983). In order to provide minimum threonine requirements homogenized milk was added, but not until the seventh day. One of the two patients deteriorated suddenly on the seventh day, with clinical manifestations similar to those observed during the first week of life. Surprisingly, CFS glycine had not increased, but there was a 16 and 19 fold elevation of threonine in CSF and plasma, respectively. We hypothesized that the unbalanced diet caused excessive protein catabolism and that the endogenous threonine load was not metabolized because of an enzymatic block. A disorder of threonine metabolism was also suggested by the finding of slightly elevated threonine and serine concentrations in plasma and CSF on four of nine tests, performed prior to the dietary treatment.

Of the three known enzymes with activity toward threonine, one was previously measured in NKH and found to be normal (Tada et al., 1969, 1974). It is serine transhydroxymethylase (STHM) (EC 2.1.2.1), which is active toward serine and threonine, also called threonine aldolase (EC 4.1.2.5) (Schirch and Gross, 1968). The other two enzymes are threonine dehydrogenase (EC 1.1.1.d) and threonine dehydratase (TD) (EC 4.2.1.16). We hypothesized (Figure 1) that deficiency of either one of these two enzymes could increase the metabolic load on STHM and that the resultant competition between threonine and serine for STHM might inhibit serine formation from glycine, causing hyperglycinaemia. The observed deficiency in glycine cleavage, which is rarely complete, thus could be a secondary phenomenon.

The original patient was no longer available for study. We therefore measured TD activity in the autopsy

Correspondence to: I. Krieger, Children's Hospital of Michigan, 3901 Beaubien, Detroit, MI 48201, USA. specimen of another patient, who is briefiy presented. TD was measured because it appears to be the enzyme most responsive to increased metabolic threonine loads (White et al., 1978) and its deficiency would thus have a more profound effect than deficiency of the dehydrogenase.

\section{MATERIALS AND METHODS}

The liver specimen was obtained within $1 \mathrm{~h}$ of death and stored at $-70^{\circ} \mathrm{C}$ for 4 months until the assays were performed. Three control specimens were stored for 1-7 days and obtained within $2-4 \mathrm{~h}$ of death. Two biopsy specimens were stored for 6 years. The control patients were four cases with congenital heart disease and one with lymphoangiomatous disease, ages 6 months to 4 years. The assay of TD was performed according to the method of Nishimura and Greenberg (1961), as modified by Yeung and Yeung (1972). The reaction product was measured by a method described by Goldstein et al. (1962). The following marker enzymes were measured: ornithine transcarbamylase (Prescott and Jones, 1969), (kindly performed by Dr Lynn Fleischer, Detroit), convertase (Mehler et al., 1958), lactate dehydrogenase (Schwartz and Bodansky, 1960), and glutamate dehydrogenase (Fahien and Cohen, 1970), (kindly performed by Dr R. A. Mitchell, Detroit).

\section{CASE DESCRIPTION}

The patient had an uneventful delivery and early neonatal course, but paucity of movements and lack of interest in food gradually became apparent. At 6 months of age, he was investigated at the Winnipeg Children's Hospital because of failure to suck, progressive hypotonia and lethargy, causing inadequate ventilation, for which he required ventilatory assistance. Myoclonic jerks and seizure activity, consisting of increased extensor tone, were observed. An electroencephalogram showed burst suppression. There was no history of vomiting. Routine laboratory investigations were negative; notably, there was no evidence of ketoacidosis, either by history or laboratory tests. However, a urine organic acid screen was not done. Amino acid analysis by 


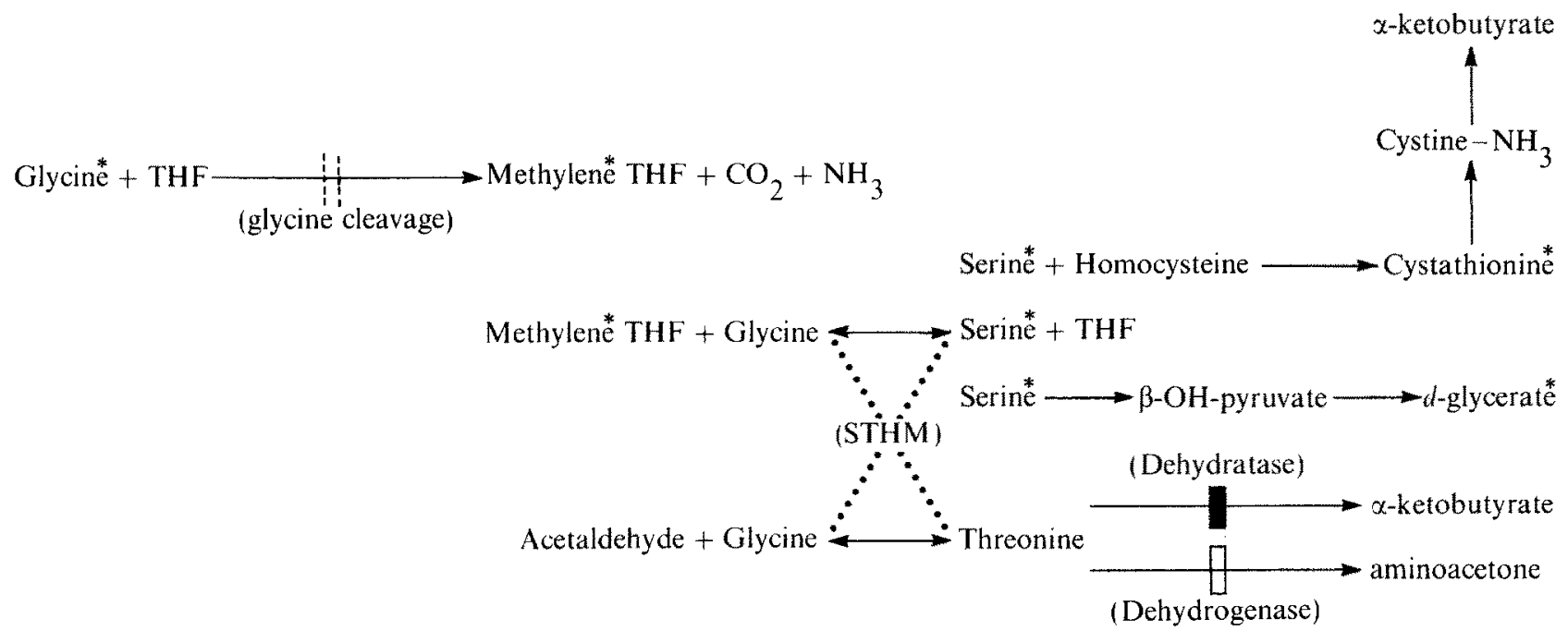

Figure 1 Non-ketotic hyperglycinaemia; proposed block and enzyme inhibition. The observed deficiency of threonine dehydratase (solid bar) or a deficiency of threonine dehydrogenase (open bar) could explain the partial deficiency of glycine cleavage (double dashed line), which has been reported in the literature, because threonine competes with glycine for serine transhydroxymethylase (dotted line). This hypothesis is compatible with observation in a previously reported case with NKH (K $\phi$ lvraa et al., 1980), where labelled glycine was administered and the label (asterisk) was observed in $d$-glycerate, with trace amounts in serine and cystathionine

column chromatography revealed elevation of plasma and cerebrospinal fluid glycine concentrations, 1920 and $370 \mu \mathrm{mol} / 1$, respectively (normal 220 and $6.6 \mu \mathrm{mol} / 1$ ). A diagnosis of NKH was made and he was placed on sodium benzoate and Clonazepam; strychnine was given in varying doses ranging from 0.5 to $1.5 \mathrm{mg} \mathrm{kg}^{-1}$ day $^{-1}$, depending on clinical condition. Within 1 week some improvement was noted, evidenced by increased muscle tone and spontaneous movements. Stopping treatment at 9 months of age was followed by increased seizure activity and failure to suck. Treatment was reinstituted, but lethargy increased 1 month later despite therapy. There was little spontaneous movement and the patient did not focus or follow. He was then placed on valium, folic acid, choline and sodium benzoate. One month later, at age 11 months, therapy was gradually discontinued, except for gavage feeding. His condition remained essentially unchanged. He withdrew to painful stimuli, but was otherwise unresponsive, with intermittent seizures consisting of flinging of the arms, eye rolling and stiffening. Sudden cardiorespiratory arrest occurred at $12 \frac{1}{2}$ months of age following a lumbar puncture which had not presented technical difficulties. Autopsy revealed petechial haemorrhages of the thyroid and pleura, and diffuse spongy changes of cerebral white matter.

\section{RESULTS}

Activity of TD was not detectable in the liver specimen of the patient; control activity was $52-107 \mu \mathrm{mol} \mathrm{h}^{-1} \mathrm{~g}^{-1}$ tissue in the three autopsy specimens and $42-156 \mu \mathrm{mol} \mathrm{h}^{-1} \mathrm{~g}^{-1}$ in the biopsy specimen. Lactate dehydrogenase was $57 \%$ and glutamate dehydrogenase $83 \%$ of controls. Convertase activity was normal. Ornithine transcarbamylase was $3974 \mu \mathrm{mol} \mathrm{h}^{-1} \mathrm{~g}^{-1}$ ti- ssue (normal: $3346 \pm 782$ ). The effect of handling was measured in rat liver. In two specimens, stored for 6 months at $-70^{\circ} \mathrm{C}$, TD activity was 112 and $113 \mu \mathrm{mol} \mathrm{h}^{-1} \mathrm{~g}^{-1}$ tissue, compared to 105 and $115 \mu \mathrm{mol} \mathrm{h}^{-1} \mathrm{~g}^{-1}$ in two fresh specimens. Following storage at $-70^{\circ} \mathrm{C}$ for 6 years, activity was only $42 \mu \mathrm{mol} \mathrm{h}^{-1} \mathrm{~g}^{-1}$. When a fresh specimen was exposed to room temperature for 2 and $4 \mathrm{~h}$, activity decreased from 105 to 96 and $87 \mu \mathrm{mol} \mathrm{h}^{-1} \mathrm{~g}^{-1}$, respectively. When the specimen that had been stored for 6 years at $-70^{\circ} \mathrm{C}$ was thawed and then exposed for $2 \mathrm{~h}$ at room temperature, activity decreased from 42 to $30 \mu \mathrm{mol} \mathrm{h}^{-1} \mathrm{~g}^{-1}$.

\section{DISCUSSION}

A disorder of threonine metabolism has not been suspected before in NKH and only one poorly documented case of hyperthreoninaemia has ever been reported (Reddi, 1978). Since it is unlikely that mutations spare consistently one amino acid, we suggest that the rarity of hyperthreoninaemia is attributable to the availability of more than one metabolic pathway. Only an excessive metabolic load will, under these circumstances, reveal evidence of a metabolic defect. Whether the deficiency of threonine dehydratase, observed by us, is indeed the primary cause of NKH must now be proven on fresh liver biopsies, although the studies in rat liver indicate that our observation is not an artifact. Moreover, the finding of normal activity of ornithine transcarbamylase-known to be an unstable enzyme-also supports our belief that the patient's specimen was viable.

Variable deficiency of glycine cleavage is the only biochemical abnormality that has been recognized in NKH to date. Similar degrees of deficiency occur in ketotic hyperglycinaemias, including methylmalonic 
and propionic acidaemia (Tada et al., 1974; Ando et al., 1972). These hyperglycinaemias are clinically and biochemically different than NKH. Deficiency of glycine cleavage has also been described in a patient with $d$ glyceric acidaemia (Kфlvraa et al., 1980) who had the clinical manifestations of NKH. Subsequently another patient with a typical clinical picture of NKH was described who also excreted large amounts of glyceric acid (Grandgeorge et al., 1980). It is not certain whether deficiency of $d$-glycerate dehydrogenase or glycine cleavage was the primary defect in these cases.

Perry et al. (1977) demonstrated complete deficiency of glycine cleavage in frozen brain tissue of five patients dying with NKH. Only one patient with ketotic hyperglycinaemia was analysed and found to be normal, while a case with an unidentified form of hyperglycinaemia had partial deficiency. In view of the small number of cases and the use of autopsy specimens, it remains questionable whether involvement of brain by the enzyme defect indeed distinguishes NKH from the ketotic hyperglycinaemias. Partial deficiency of glycine cleavage in liver of cases with ketotic hyperglycinaemia is recognized as a secondary phenomenon. Deficiency of glycine cleavage thus cannot be used in the diagnosis of NKH. Elevation of CSF glycine and absence of ketoacidosis is currently the only distinctive feature of NKH. Prenatal diagnosis may be attempted by demonstrating glycine elevation in amniotic fluid (Garcia-Castro et al., 1982), but the reliability of this approach remains to be proven. Demonstration of a specific enzyme defect in NKH thus may provide a more reliable method for prenatal diagnosis, if it can be shown that the enzyme is present in amniotic fibroblasts.

It is possible that the described deficiency of TD is secondary to inhibition by glycine or by an unknown intermediary metabolite. However, the reverse is a more likely explanation since the TD defect was complete, whereas substantial glycine cleavage activity remained in most of the reported cases. The reverse explanation is compatible with current concepts of intermediary threonine metabolism. Since the affinity of STHM is greatest for serine and lowest for threonine, competition between glycine and threonine can be anticipated when there is a block of threonine catabolism due to deficiency of either, threonine dehydratase or threonine dehydrogenase (Figure 1). This explanation is also supported by the occasional finding of slightly elevated levels of plasma serine and threonine in our original patient. Using labelled glycine, Kфlvraa et al. (1980) showed that, aside from $d$-glyceric acid, trace amounts of the label appear in serine and cystathionine. This observation is compatible with our hypothesis. Appearance of the label in cystathionine explains the elevation of $\alpha$ aminobutyric acid, which was observed at the time of hyperthreoninaemia in the original case, although it was not included in the report (Krieger and Nigro, 1983). This metabolite is derived from $\alpha$-ketobutyric acid. Since $\alpha$-ketobutyrate derives from both, serine as well as threonine, it is possible that threonine dehydrogenase was defective in this original case.

Our hypothesis also explains the finding of $d$-glycerate in the patient of Kфlvraa et al. (1976) who showed clinical manifestations of NKH but had, in addition, glycerate dehydrogenase activity in the heterozygous range. This amount of enzyme function should not cause accumulation of $d$-glycerate unless there is an excessive load of serine, which is not readily converted to glycine. We hypothesize that this occurs in NKH because of the competition between serine, threonine and glycine for STHM. Mutations appear to be common in man, most of which are silent because of heterozygosity of recessive traits or because compensatory pathways exist. One may therefore anticipate that heterozygous mutations can co-exist with other inborn errors of metabolism. Partial deficiency of glycerate dehydrogenase, as observed by Kolvraa et al. (1976), would be biochemically 'silent' unless the load is excessive. Co-existence of this defect thus may have been a chance occurrence.

MS received 14.6 .83

Accepted for publication 19.8 .83

\section{References}

Ando, T., Nyhan, W. L, Connor, J. D., Rasmussen, K., Donnell, G., Barnes, N., Cottom, D. and Hull, D. The oxidations of glycine and propionic acid in propionic acidemia with ketotic hyperglycinemia. Pediatr. Res. 6 (1972) 576-583

Fahien, L. A. and Cohen, P. P. L-Glutamate dehydrogenase (frog and tadpole liver). Methods Enzymol. 17A (1970) 839

Garcia-Castro, J. M., Isales-Forsythe, C. M., Levy, H. L., Shih, V. E., Laó-Vélez, C. R., González-Ríos, M. D. C. and Reyes de Torres, L. C. Prenatal diagnosis of non-ketotic hyperglycinemia. N. Engl. J. Med. 306 (1982) 79-81

Goldstein, L., Knox, E. W., and Behrman, E. J. Studies on the nature, inducibility, and assay of the threonine and serine dehydrase activities of rat liver. J. Biol. Chem. 237 (1962) $2855-2860$

Grandgeorge, D., Favier, A., Bost, M., Frappat, P., Boujet, C., Garrel, S., and Stoebner, P. L'acidémie D-glycérique; A propos d'une nouvelle observation anatomo-clinique. Arch. Fr. Pediatr. 37 (1980) 577-584

Kфlvraa, S., Christensen, E. and Brandt, N. J. Studies of the glycine metabolism in a patient with $d$-glyceric acidemia and hyperglycinemia. Pediatr. Res. 14 (1980) 1029-1035

Kølvraa, S., Rasmussen, K. and Brandt, N. J. D-Glyceric acidemia. Biochemical studies in a new syndrome. J. Pediatr. Res. 10 (1976) 825-828

Krieger, I. and Nigro, M. Evidence for defective threonine metabolism in nonketotic hyperglycinaemia. $J$. Inher. Metab. Dis. 6 (1983) 40-43

Maher, J. T. and Wurtman, R. J. L-Threonine administration increases glycine concentration in the rat central nervous system. Life Sci. 26 (1980) 1283-1286

Mehler, A. H., McDaniel, E. G., and Hundley, J. M. Changes in the enzymatic composition of the liver, J. Biol. Chem. 232 (1958) 323-330

Nishimura, J. S. and Greenberg, D. M. Purification and properties of $\mathrm{L}$-threonine dehydrase of sheep liver. J. Biol. Chem. 236 (1961) 2684-2691

Perry, T. L., Urquhart, N., Hansen, S, and Mamer, O. A. Studies of the glycine cleavage enzyme system in brain from infants with glycine encephalopathy. Pediatr. Res. 12 (1977) 1192-1197

Prescott, L. M. and Jones, M. E. Modified methods for the determination of carbamyl aspartate. Anal. Biochem. 32 (1969) 408-419 
Reddi, O.S. Threoninemia - a new metabolic defect. $J$. Pediatr. 93 (1978) 814-816

Schirch, L. and Gross, T. Serine transhydroxymethylase identification as the threonine and allothreonine aldolases. J. Biol. Chem. 243 (1968) 5651-5655

Schwartz, M. K. and Bodansky, O. Lactate dehydrogenase (clinical aspects). Methods Enzymol. 9 (1960) 294

Tada, K., Narisawa, K., Yoshida, T., Konno, T., Yokayama, Y,, Nakagawa, H., Tanno, K., Mochizuki, K. and Arakawa, T. Hyperglycinemia: a defect in glycine cleavage reaction. Tohoku J. Exp. Med. 98 (1969) 289-296
Tada, K., Corbeel, L. M., Eeckels, R. and Eggermont, E. A block in glycine cleavage reaction as a common mechanism in ketotic and nonketotic hyperglycinemia. Pediatr. Res. 8 (1974) 721

White, A., Handler, P., Smith, E. L., Hill, R. L. and Lehman, T. R. Principles of Biochemistry. McGraw-Hill, New York, 1978, p. 737

Yeung, Y. G. and Yeung, D. The postnatal induction of threonine dehydratase in rat liver. J. Biochem. 3 (1972) $180-186$

\section{Case Report}

\section{PINK NAPKINS - PRESENTING FEATURE IN A CASE OF ALKAPTONURIA}

\section{A. Sutherland ${ }^{1}$, A. D. Nicol ${ }^{1,2}$ and A. J. Williams ${ }^{1}$}

Pink staining of an infant's napkin is commonly attributed to uric acid crystals in urine whilst other, rarer causes include haemoglobinuria, dyes from sweets, anthocyanins from beetroot or berries, drugs, porphyria and contamination with Serratia marcescens (Cone, 1968). We report a case of alkaptonuria (McKusick 20350) presenting with pink staining of the napkin.

E.E. is a healthy, well-developed boy, the result of a normal pregnancy; his sister is 3 years older and is well. E.E. was referred at age 8 weeks after his mother reported pink-stained napkins to the Health Visitor. Discolorations had been noticed from age 1 week (washing both napkins and baby's buttocks produced a deep brown colour). Vitamin supplement administration coincided with disappearance of staining, which returned on discontinuing vitamins. Maternal vitamin C administration yielded unstained napkins but these became pink again when breast-feeding ceased. Additional vitamin C in E.E.'s diet keeps his napkins discoloration-free.

Urine samples were generally clear, of normal $\mathrm{pH}$, and did not exhibit suspicious darkening. They were positive for reducing substances, negative for glucose, giving a transient blue-green colour with ferric chloride. Following alkalinization, urine darkened rapidly from the meniscus downwards, becoming very dark brown within hours. Alkaptonuria was confirmed as follows.

\footnotetext{
${ }^{1}$ Departments of Paediatrics and Chemical Pathology, Glan Clwyd Hospital, Bodelwyddan, Clwyd LL18 5UJ, UK

${ }^{2}$ Corresponding author
}

Spectroscopic examination of urine diluted in $0.2 \mathrm{~mol} / \mathrm{l}$ phosphate buffer $\mathrm{pH} 6.5$ revealed the presence of large quantities of a substance with an absorption maximum at $290.2 \mathrm{~nm}$ : the absorption maximum for homogentisic acid is $290 \mathrm{~nm}$ at $\mathrm{pH}$ 6.8. Under similar conditions uric acid has a maximum at $291 \mathrm{~nm}$. However, derivative spectra, namely, $\frac{\mathrm{d}^{2} \mathrm{~A}}{\mathrm{~d} \lambda^{2}}$ (Fell, 1981), from dilutions of E.E.'s urine were quite different from those of control urines. Furthermore, the maximum at $290.2 \mathrm{~nm}$ rapidly disappeared on treating E.E.'s urine with horse-radish peroxidase and glutathione ( $\mathrm{La} \mathrm{Du}$ and Zannoni, 1963), whereas uricase effected little change. By contrast, control urines with absorption maxima around $293 \mathrm{~nm}$ behaved in the converse manner. Finally, gas-liquid chromatography of E.E.'s urinary organic acids yielded a very large peak which cochromatographed with authentic homogentisic acid (Dr. D. Isherwood, Alder Hey Hospital, Liverpool).

Aqueous homogentisic acid at $\mathrm{pH} 6$ turns pink in daylight. In 1929 Garrod observed red-coloured urine in alkaptonuric subjects. Thus it is to be expected that alkaptonuria may reveal itself by pink staining of napkins and not exclusively by the traditional brown discoloration.

\section{References}

Cone, T. E. Diagnosis and treatment: Some syndromes, diseases and conditions associated with abnormal coloration of the urine or diaper. J. Pediatr. 41 (1968) 654 658

Fell, A. F. Derivative spectroscopy in the analysis of aromatic amino acids. In Rattenbury, J. M. (ed.) Amino Acid Analysis. Ellis Horwood, Chichester, 1981, pp 86-118

La Du, B. N. and Zannoni, V. G. Oxidation of homogentisic acid catalysed by horse-radish peroxidase. Biochim. Biophys. Acta 67 (1963) 281-287 